\title{
Contraintes et libertés dans le passage à l'anglais du discours mathématique
}

\section{Michel Petit}

\section{CpenEdition}

\section{Journals}

Édition électronique

URL : http://journals.openedition.org/asp/4274

DOI : $10.4000 /$ asp.4274

ISSN : 2108-6354

\section{Éditeur}

Groupe d'étude et de recherche en anglais de spécialité

\section{Édition imprimée}

Date de publication : 1 octobre 1993

Pagination : 93-106

ISSN : 1246-8185

\section{Référence électronique}

Michel Petit, «Contraintes et libertés dans le passage à l'anglais du discours mathématique », ASp [En ligne], 2 | 1993, mis en ligne le 27 février 2014, consulté le 01 mai 2019. URL : http:// journals.openedition.org/asp/4274 ; DOI : 10.4000/asp.4274

Ce document a été généré automatiquement le 1 mai 2019.

Tous droits réservés 


\title{
Contraintes et libertés dans le passage à l'anglais du discours mathématique
}

\author{
Michel Petit
}

L'objet de cette communication est de mettre en évidence certaines des contraintes linguistiques qui s'exercent sur le passage à l'anglais du discours mathématique et des libertés qui y sont permises. C'est dire qu'elle se place résolument dans une perspective interlinguistique, qu'il s'agisse de traduction en anglais de textes mathématiques rédigés en français, ou qu'il s'agisse de rédaction directe en anglais d'un texte mathématique par un locuteur francophone ${ }^{1}$. On peut en effet, semble-t-il, parler dans ce dernier cas de passage à l'anglais du discours mathématique, même si ce discours n'est pas matérialisé par un texte en français, dans la mesure où le locuteur francophone, non bilingue et non spécialiste d'anglistique, se trouve nécessairement sous l'influence des structures (pour faire bref et non technique sur un point complexe) de sa langue maternelle. Il n'est donc pas étonnant de parler ici de contraintes, la traduction, ou le passage à l'anglais, consistant en effet à assurer le passage d'un système linguistique avec son ensemble de contraintes spécifiques, à un autre système linguistique également défini par un ensemble de contraintes dont la nature et l'organisation font qu'il ne correspond pas exactement, c'est-à-dire terme à terme, au système de la langue de départ. Le discours mathématique ne fait pas en ce sens exception à la règle : les contraintes linguistiques sur son passage à l'anglais résultent de ce que le français et l'anglais ont un fonctionnement différent. On peut se demander en revanche quelles y sont les libertés. Si en effet la traduction littéraire comporte toujours, nécessairement, une composante de choix stylistiques, le discours mathématique paraît présenter un type de fonctionnement discursif dont le style relève plus de la typologie des discours ${ }^{2}$ que de choix individuels, ce qui semble réduire, dans la perspective interlinguistique, sinon la possibilité du moins la nécessité des choix stylistiques. Le style des textes mathématiques en anglais est en ce sens largement prédéterminé par leur appartenance au type de discours mathématique. À l'intérieur même de ce discours, on peut reconnaître plusieurs niveaux, souvent identifiables au caractère stéréotypé de leur formulation, et l'analyse conduit en particulier à distinguer une fonction de commentaire et une fonction de démonstration ${ }^{3}$. 
Ainsi, pour résumer la problématique telle qu'elle apparait à ce stade, le passage à l'anglais du discours mathématique peut être perçu comme soumis à deux ensembles de contraintes: celles, fortes, qui tiennent au fonctionnement de la langue et dont la transgression entraîne des énoncés incorrects, et celles qui tiennent au fonctionnement du type de discours et dont le non-respect entraîne des énoncés moins "naturels » ou moins authentiques. C'est cette conception qui sous-tend l'approche illustrée par l'excellent ouvrage de Marc Défourneaux, Do you speak science?, dont une partie vise à présenter « la spécificité d'un certain "style scientifique" qui tient de l'usage et non de la seule grammaire, avec des expressions et des locutions parfois déroutantes pour les francophones » (Défourneaux 1980 : xiii). Mais quelle que soit sa valeur pratique pour le francophone rédigeant en anglais, qui voit ainsi son attention attirée sur un certain nombre de contraintes donnant lieu à des divergences entre les deux langues et de contraintes plus diffuses tenant au type de discours, cette approche laisse inexploré le domaine des libertés et des contraintes énonciatives ${ }^{4}$. Le problème se pose de façon particulièrement nette dans le cas de certaines configurations françaises, récurrentes dans le discours mathématique en français, qui sont sans équivalent direct en anglais. Ainsi, l'absence d'un équivalent exact de "on » en anglais entraîne pour chacune des occurrences de ce marqueur la nécessité d'opérer un choix de traduction entre les diverses formulations possibles. Cette liberté nécessaire est celle du champ énonciatif, et de sa nécessaire dimension discursive, et on voit qu'elle s'offre (ou s'impose) tout autant à l'anglophone traduisant dans sa langue un texte français qu'au francophone rédigeant son texte en anglais. Ce sont donc ces contraintes et ces divers degrés de liberté énonciative dont nous nous proposons d'analyser l'interaction à travers l'approche contrastive de trois cas précis, les verbes pronominaux, le marqueur « on » et l'impératif français de première personne, représentatifs des phénomènes de passage à l'anglais du discours mathématique français ${ }^{5}$.

\section{Les verbes pronominaux français}

Il s'agit d'un problème de traduction classique, qui résulte de l'existence en français d'une classe spécifique de verbes sans équivalent direct en anglais. Le discours mathématique en offre, comme le français en général, un grand nombre d'exemples: "nous nous bornerons à considérer... », « on se limitera à l'étude de... », " on se servira alors de... », «il est clair que la fonction s'applique à... », «le résultat se ramène alors à...", «la solution se réduit à... », etc. On voit que ces verbes, tout en relevant du même statut de verbe pronominal, présentent des caractéristiques d'emploi différentes, tenant à la nature du sujet (pronom personnel ou indéfini, objet mathématique) et à leur nature sémantique. Il convient donc, avant de s'intéresser aux conditions de leur traduction, de les examiner dans une perspective intralinguistique. On peut à cet égard caractériser les verbes pronominaux français de façon formelle par deux traits distinctifs essentiels : « la présence d'un pronom conjoint de même rang que le sujet : "je me, tu te, il se", etc. », et "l'emploi constant de l'auxiliaire "être" aux temps composés » (Gardes-Tamine 1990 : 86). Si les auteurs semblent aujourd'hui s'accorder à reconnaître que les verbes pronominaux ne constituent pas, à côté de l'actif et du passif, une troisième voix ${ }^{6}$, une certaine diversité règne dans la littérature quant à leur classification en catégories, où l'on peut néanmoins distinguer les quatre types suivants : verbes réfléchis (par exemple, « se laver », et dans le discours mathématique, «se borner », " s'appliquer »), verbes réciproques ("se battre », 
et «se couper»: «les droites se coupent»), verbes essentiellement pronominaux ("s'évanouir, se repentir», et "se servir de»), verbes de sens passif ("se manger, se vendre », et "s'obtenir», "s'expliquer»). C'est généralement à partir de cette classification sémantique, en rappelant la diversité des «sens » possibles de la forme pronominale en français, que les grammaires françaises de l'anglais traitent de la traduction des verbes pronominaux :

Attention. Il faut bien comprendre l'interprétation à donner à la construction pronominale. Le passage à l'anglais n'est pas automatique, et souvent le pronom français « se » n’a aucun équivalent en anglais. (Larreya \& Rivière 1991 : 222)

On voit donc que la contrainte principale pesant sur le passage à l'anglais des verbes pronominaux résulte bien de l'absence d'une classe de verbes anglais équivalents, c'est-àdire de l'absence d'un marqueur unique toujours équivalent au «se » français, puisque c'est ce marqueur qui est l'élément constitutif de la définition. Il existe en revanche en anglais des pronoms réfléchis et des pronoms réciproques, qui peuvent donc correspondre respectivement au «se » français des verbes de sens réfléchi et de sens réciproque. Mais leur emploi n'est pas automatique. Ainsi, le tableau récapitulatif de la Grammaire explicative de l'anglais (Larreya \& Rivière 1991 : 222-223) fait apparaître, pour la traduction des verbes de sens réfléchi, les deux cas suivants : « objet réfléchi non exprimé en anglais » ("Elle se lave. She is washing.»), ou " pronom réfléchi en -self» ("Elle s'est blessée. She has hurt herself.»). De la même façon, la traduction des verbes de sens réciproque peut comporter un "effacement de l'objet réciproque " ou l'emploi d'un " pronom réciproque each other ou one another. » Cette liberté est elle-même soumise à un certain nombre de contraintes tenant par exemple au caractère marqué des pronoms réfléchis anglais, ce que nous allons maintenant nous attacher à démontrer.

\section{Traduction d'un verbe pronominal français par un verbe réfléchi anglais}

4 L'existence de pronoms réfléchis en anglais permet une construction réfléchie des verbes de sens réfléchi, parmi lesquels on peut distinguer des

obligatorily reflexive verbs, i.e. verbs which always require reflexive object, such as absent oneself (from), avail oneself (off, betake oneself, pride oneself (on) et des

optionally reflexive verbs, i.e. verbs where the reflexive pronoun may be left out with little or no change in meaning, such as adjust (oneself), dress (oneself), prove (oneself to be competent), shave (oneself), wash (oneself) (Quirk et al. 1972 : 211)

Cette distinction paraît peu productive dans le cas qui nous occupe, puisque la nature des procès de type réfléchi observables dans le discours mathématique français permet de noter une certaine spécialisation lexicale, qui exclut généralement les verbes désignant des actes concrets de la vie quotidienne («se laver », etc.) au profit de verbes relevant de la fonction de commentaire mathématique («se donner des conditions, se limiter à, se borner à ») ou de la fonction de démonstration (" s'appliquer, se réduire, s'adapter »). Ces deux catégories, commentaire et démonstration, ne sont pas également compatibles avec la valeur centrale de la forme réfléchie anglaise, telle qu'elle ressort de l'analyse suivante :

Pour que la forme réfléchie puisse être employée en anglais, il faut non seulement que l'action revienne sur le complément qui est la même personne ou la même chose que le sujet, mais aussi que l'action soit faite volontairement par le sujet sur 
lui-même... Cela revient à dire que si le sujet est incapable de détermination, si l'action est involontaire, ou encore familière au point de ne pas entraîner l'intervention de la volonté, on n'emploiera pas la forme réfléchie. (Faure \&

Casanova $1968: 122)$

6 On voit facilement que seuls les verbes de commentaire, généralement employés avec des sujets personnels (« nous » ou « on » lorsqu'il est équivalent à « nous »), souvent dans des contextes futurs, sont parfaitement compatibles avec la traduction par une forme réfléchie qui ne soulève donc aucune difficulté particulière. Les traductions autres que réfléchies que l'on peut observer pour ce type de verbes résultent donc d'une liberté stylistique, dont le but est souvent d'alléger le style en évitant par exemple l'utilisation trop rapprochée de pronoms réfléchis, perçus comme "des formes lourdes et incommodes » (Faure \& Casanova $1968: 120$ ). En ce qui concerne les verbes réfléchis de démonstration, la nature du sujet grammatical qui est généralement un objet mathématique les rend incompatibles avec la valeur centrale des réfléchis anglais. La traduction aura donc le plus souvent recours à un verbe lexical simple, comme nous allons le voir.

\section{Traduction d'un verbe pronominal par un verbe lexical simple en anglais}

7 Elle s'observe d'abord dans le cas évoqué ci-dessus. M. Défourneaux, à partir de la phrase "La formule s'applique à...", signale que, "en anglais, le verbe to apply signifie indifféremment "appliquer" ou bien "s'appliquer", et il en est de même de nombreux verbes d'emploi courant en langage scientifique", dont il donne une liste en précisant que «en cas de doute sur d'autres verbes, il faut consulter un dictionnaire " (Défourneaux 1980: 79). On la trouve également pour un certain nombre de verbes, de sujet personnel («Nous nous servirons de..», "On se souvient que...»), impersonnel ("d'où il s'ensuit que..»), ou mathématique («Le résultat s'ensuit»), relevant de la catégorie des verbes essentiellement pronominaux. Ces verbes, à l'exception de "s'ensuivre ", paraissent moins nombreux dans le discours mathématique que dans les utilisations non spécialisées du français ${ }^{7}$. Leur forme est celle d'

une expression entièrement lexicalisée dont le rapport avec le verbe simple n'est pas descriptible (Gardes-Tamine $1990: 87$ )

8 On ne peut pas opposer le pronominal à un verbe simple (par exemple *ensuivre). On peut donc dire que

les pronoms réfléchis qui les accompagnent ont perdu le sens réfléchi, et ils servent uniquement à attirer l'attention sur le sujet. Très logiquement, l'anglais les négligera et le verbe français sera rendu par la voix active. (Faure \& Casanova 1968 : 123)

\section{Traduction d'un verbe pronominal par un verbe au passif}

M. Défourneaux signale cette traduction, à partir de l'expression «L'équation se résout en... », en proposant le commentaire suivant :

en anglais, on considère que ce n'est pas l'équation qui se résout elle-même, mais qu'on la résout, ce qui se traduit par la forme passive (Défourneaux 1980 : 79)

ce qui est une autre façon de dire que le réfléchi est ici impossible, pour les raisons qu'on a déjà vues. Quant à la glose par « on », elle nous paraît compliquer l'explication, car elle introduit, par rapport au verbe pronominal, un changement d'orientation de la relation, 
changement que la traduction anglaise n'entraîne pas. La traduction d'un pronominal de sens passif par une structure passive est en effet très directe puisque la relation prédicative y conserve le même sujet, qui est généralement un objet mathématique. Elle est donc parfaitement adaptée aux caractéristiques de dépersonnalisation du discours mathématique. On notera que les pronominaux concernés, "s'écrire, s'exprimer, s'obtenir, s'expliquer, s'interpréter...» semblent souvent inclure, même à des formes simples et apparemment non marquées comme le présent, une dimension modale, que la traduction anglaise est conduite à expliciter. Ainsi, la phrase « Le résultat se réécrit alors sous la forme... » se traduit bien par "The result can then be rewritten as... » (et non pas " The result is then rewritten as...»). La traduction retrouve ici les contraintes plus générales de la langue, telles que le fonctionnement de la modalitée

\section{Le pronom français « on »}

Il s'agit là encore d'un problème de traduction tout à fait classique, auquel s'intéressent nombre de grammaires françaises de l'anglais et la plupart des manuels de traduction. La contrainte résulte ici à nouveau de l'absence en anglais d'un marqueur unique toujours équivalent à "on", dont on connaît bien la diversité des emplois et des valeurs en français :

il traduit la notion de personne sous un aspect indéterminé, et il peut se substituer

à des pronoms de différente personne. (Wagner \& Pinchon $1962: 203$ )

Le débat sur son statut de pronom personnel ou de pronom indéfini tient en grande partie à cette flexibilité qui lui permet à la fois de fonctionner comme marqueur de référence spécifique, c'est-à-dire de désigner une ou plusieurs personnes déterminées (comme le ferait un pronom personnel), et comme marqueur de référence générique, indéterminé quant à la personne. C'est également là le point central des conditions de son passage à l'anglais :

Pour traduire le pronom français « on", il faut commencer par le ramener à son

sens véritable (Faure \& Casanova $1968: 137$ ),

c'est-à-dire déterminer sa valeur énonciative, qui peut aller de l'indétermination maximale à des formes de détermination partielle excluant ou incluant l'énonciateur et le co-énonciateur (Chuquet \& Paillard 1989: 67-70). Cette caractéristique de «on» qui permet de réduire les interventions explicites de l'énonciateur dans un discours dont le caractère scientifique va de pair avec la dépersonnalisation, ajoutée au caractère économique de «on" sur le plan morphologique et syntaxique, explique sa fréquence élevée dans le discours mathématique. Nous allons examiner rapidement les contraintes et les libertés à l'œuvre dans ses principales traductions en anglais.

\section{Traduction de « on » par «we »}

14 C'est de loin la traduction la plus fréquente, et par exemple la traduction quasiment unique de l'omniprésent «on a " =we have». Aussi économique sur le plan morphologique que le «on » français, elle ne présente qu'une contrainte énonciative : l'inclusion de l'énonciateur. Elle correspond en ce sens parfaitement à tous les emplois où " on » pourrait être remplacé en français par «nous », que ce " nous » soit lui-même un «nous de modestie » équivalent à «je » («On montrera dans le chapitre $\mathrm{X}$ ») ou cette forme de «nous d'auteur» qui inclut le lecteur dans la démarche scientifique («on 
aboutit ainsi à... »). On la trouve tout à la fois pour les procès relevant de la fonction de commentaire (" on verra dans la section suivante " = we shall see in the following section ») 9 et de la fonction de démonstration ( on a alors " = "we then have »). Il faut noter que, dans l'autre sens, le sens anglais-français, le traducteur a donc pour ce type d'énoncés toujours la liberté de choisir de traduire le «we » anglais par un « on » ou un «nous » en français.

\section{Traduction de « on » + syntagme verbal par un passif en anglais}

Elle est moins fréquente dans le discours mathématique que dans d'autres types d'utilisation de la langue, peut-être parce que le passif y est déjà largement sollicité pour d'autres formulations récurrentes (verbes pronominaux par exemple). On la trouve surtout avec des verbes définitoires : " on dit que », " on appelle », etc. La nature et la composition de ces énoncés font apparaître des contraintes syntaxiques spécifiques en anglais, comme on le voit en examinant l'énoncé suivant ${ }^{10}$.

on appelle structure de g-module sur V la donnée d'un homomorphisme d'algèbres de Lie $\emptyset: \mathrm{g} \rightarrow$ End $(\mathrm{V})$

a Lie algebra homomorphism $\varnothing: \mathrm{g} \rightarrow$ End $(\mathrm{V})$ is called a g-module structure on $\mathrm{V}$

La principale modification observée tient à la place du verbe dans la structure de la phrase. Alors que le français présente une structure syntaxique caractérisée par la présence à droite $\mathrm{du}$ verbe de deux groupes nominaux complexes qui sont respectivement, dans l'ordre linéaire, attribut ("structure de g-module sur V») et complément (« la donnée d'un homomorphisme d'algèbres de Lie $\varnothing: \mathrm{g} \rightarrow$ End $(V) »)$, le complément correspondant à l'élément appelé et l'attribut à l'élément appellateur, la traduction anglaise présente une structure canonique sujet-verbe-attribut. Si dans la phrase française la fonction de chacun des deux groupes n'est pas déterminée par leur place sur la chaîne (on pourrait en effet sans changement de sens en inverser l'ordre), il est clair que l'organisation de la phrase anglaise, notamment la place de chaque élément par rapport au verbe, est un élément constitutif du sens, l'attribut ne pouvant en effet se trouver qu'à droite du verbe. C'est en français la présence d'un marqueur de détermination nominale, article défini pour le complément, article zéro pour l'attribut, qui constitue le critère déterminant pour l'interprétation de la construction, alors que cette détermination nominale obéit en anglais à d'autres règles plus générales, comme on le voit dans le groupe attribut. L'ensemble de ces phénomènes permet de percevoir pourquoi le recours à une structure passive est la solution unique pour la traduction de ce type d'énoncés.

\section{Traduction de « on » par « one »}

Elle est relativement rare dans le discours mathématique où elle correspond essentiellement à certains emplois relevant d'un statut textuel déterminé dans l'organisation discursive: énoncés de théorèmes, de lemmes, de définitions, etc. On retrouve donc les valeurs classiques de " one » ainsi que son caractère formel, c'est-à-dire ce qui fait également qu'on le trouve dans les énoncés de proverbes, de préceptes moraux, etc. On peut en outre identifier des contraintes contextuelles dont on verra une illustration en reprenant l'exemple déjà partiellement cité plus haut : 
On peut reformuler le théorème ci-dessus en termes de g-modules. Rappelons pour cela que, si g est une algèbre de Lie et $\mathrm{V}$ un espace vectoriel, on appelle structure de g-module sur $\mathrm{V}$ la donnée d'un homomorphisme d'algèbres de Lie $\emptyset: \mathrm{g} \rightarrow$ End $(\mathrm{V})$; on dit aussi que $\varnothing$ est une représentation linéaire de $\mathrm{g}$ dans $\mathrm{V}$.

We can reformulate the above theorem in terms of g-modules. To do this, we recall that if $\mathrm{g}$ is a Lie algebra and $\mathrm{V}$ a vector space, then a Lie algebra homomorphism Lie $\varnothing: \mathrm{g} \rightarrow$ End $(\mathrm{V})$ is called a g-module structure on V; one also says that $\varnothing$ is a linear representation of $\mathrm{g}$ on $\mathrm{V}$.

Il paraît clair que la traduction de « on dit aussi que Ø... » par un passif, «䥚 is also said to be... ", poserait ici un certain nombre de difficultés syntaxiques et contextuelles, tenant notamment à la présence de l'adverbe « aussi ». La traduction par « we also say » créerait, elle, un parallélisme avec le «we » du début de l'énoncé, qui relève manifestement d'un autre niveau d'argumentation. "One » est donc une solution pratique et compatible à la fois avec les contraintes énonciatives et contextuelles.

\section{Traduction de « on » + syntagme verbal par un impératif en anglais}

Elle correspond en général à une série de verbes tels que: "supposer, noter, poser, remarquer, observer...». Il s'agit de verbes que l'on trouve concurremment et fréquemment dans le discours mathématique sous la forme d'impératifs : « on suppose » et "supposons", dont la traduction peut également être «suppose ». Nous reviendrons dans la section suivante sur l'impératif, mais on voit que tout tourne ici autour de la valeur énonciative du " on ", valeur déterminée par un ensemble de paramètres incluant notamment le sémantisme du verbe et son temps, ainsi que le contexte. Examinons l'ensemble de ces points. Si l'on se réfère à ce qui a été dit plus haut sur la traduction de « on » par « we », la traduction la plus immédiate de « on suppose » est « we suppose », les deux marqueurs ayant la même valeur énonciative de référence globale à l'ensemble non distingué de l'auteur et de ses lecteurs. La traduction par «suppose » semble privilégier une interprétation énonciative qui exclut l'auteur, puisque l'impératif anglais est traditionnellement perçu comme une deuxième personne. Pour simplifier (peut-être à l'excès), on pourrait dire que la charge de la supposition est laissée au lecteur. Il y a donc ici emploi, non contraint, d'une forme qui correspond à un choix énonciatif de mise en valeur de la relation intersubjective. C'est encore un phénomène touchant à la relation intersubjective qu'illustre la traduction fréquente de «On rappelle que...» par « Recall that ». On voit qu'il y a là, en relation avec le sémantisme du verbe en français et en anglais, une inversion de ce que les ouvrages de traduction appellent souvent le point de vue : l'auteur "rappelle que », c'est-à-dire que les lecteurs doivent «se rappeler que ». Pour ne pas multiplier les exemples, contentons-nous de signaler le cas de certains verbes au futur, tels que "on remarquera, on consultera, on se reportera", où l'ensemble des critères indique clairement que la valeur énonciative de «on» est exclusivement de référence au lecteur. La traduction par un impératif, «notice, consult, see » n'y correspond plus à l'exercice d'une liberté énonciative mais bien à une forme de contrainte énonciative. 


\section{L'impératif français}

21 À la différence des deux points étudiés jusqu'ici, la traduction de l'impératif français n'est pas un problème classique de traduction. S'il est vrai que l'anglais ne présente pas de forme simple pour l'expression de l'impératif de première personne, où il a donc recours à une tournure utilisant l'opérateur «let », l'équivalence ne pose, dans les utilisations courantes de la langue ("partons» = «let's go »), aucune difficulté. Pour la deuxième personne, l'équivalence est encore plus directe, puisqu'il existe en anglais une forme simple d'impératif, représentée par la base verbale nue. Or, le discours mathématique présente une particularité remarquable de passage à l'anglais :

En français, généralement en début de phrase, on emploie souvent l'impératif à la première personne du pluriel. En anglais, ceci se traduit aussi par un impératif, mais à la deuxième personne. (Défourneaux 1980 : 78)

22 Ainsi, «supposons, posons, considérons, écrivons...» se traduiront souvent respectivement par «suppose, put, consider, write...». Ce phénomène paraît limité aux emplois spécialisés de la langue, discours mathématique et plus généralement tout discours de démonstration, notamment scientifique (sciences physiques par exemple). On ne trouve pas d'équivalent dans la langue courante, à l'exception de l'emploi de "'say', dans le sens de "let us say" (“disons, mettons") » (Berland-Delépine 1989 : 210). Il semble bien que dans ce cas la valeur de ce syntagme tienne précisément à ce qu'il entraîne une implication du co-énonciateur dans l'approximation ou l'estimation introduites par « say ». Nous allons voir que cette traduction de l'impératif français de première personne par un impératif anglais n'est jamais obligatoire, même si elle est souvent la traduction par défaut, celle que l'on applique lorsqu'aucune contrainte ne l'interdit et qu'aucune indication ne rend une autre traduction préférable.

\section{Contraintes interdisant la traduction de l'impératif français par un impératif en anglais}

Il s'agit essentiellement de contraintes énonciatives résultant du sémantisme des procès concernés. Ainsi, l'impératif «montrons » ne saurait être traduit par «show», puisque le sens de ce syntagme ne peut correspondre en anglais qu'à une véritable injonction adressée au lecteur. Il en va de même des verbes " prouvons ", « établissons », etc. Il n'y a plus dans ce cas de démonstration partagée entre l'auteur, qui la rédige, et le lecteur, qui la suit en s'en appropriant les étapes, mais une instruction donnée au seul lecteur, ce qui correspond alors à un énoncé d'exercice. On notera que, dans les ouvrages français relevant du discours mathématique de niveau universitaire, les énoncés d'exercices font l'objet d'une formulation à l'infinitif, dont la valeur injonctive apparaît alors clairement : «montrer, prouver, établir... ». Il est significatif que la forme utilisée en anglais, la base verbale nue, soit précisément une forme commune à l'impératif et à l'infinitif, ainsi qu'au subjonctif, subjonctif dont on sait par ailleurs qu'il est en français la forme de substitution de l'impératif aux personnes où celui-ci n'existe pas. Formes et valeurs se trouvent ainsi au cœur de la modalité et de la relation intersubjective. Indiquons simplement pour terminer sur ce facteur sémantique que la valeur de ces procès est tellement forte que la contrainte résultant de la présence d'un tel verbe dans un énoncé s'exerce sur d'autres éléments de l'énoncé. Ainsi, dans l'exemple suivant: 
Pour établir que... remarquons que...

24 l'impératif ne peut être traduit par «notice », qui serait, du fait de la relation syntaxique avec «pour établir que ", perçu comme une deuxième personne et correspondrait alors, par l'exclusion de l'énonciateur, à une injonction véritable (par exemple énoncé d'exercice). La traduction doit donc expliciter la première personne pour rétablir l'énonciateur :

To establish that..., we remark that...

To establish that..., let us notice that...

\section{Les autres traductions de l'impératif français}

Il s'agit donc, comme on l'a vu avec l'exemple ci-dessus, essentiellement de la traduction par une locution impérative avec l'opérateur « let » (" Montrons ensuite = Let us then show ») et de la traduction par un syntagme verbal de première personne pluriel au présent simple ( Commençons par = We begin with »), Elle inclut souvent l'apparition d'un modal en anglais ("Ecrivons alors que... = Then we may write that...»), ce qui conforte les observations précédentes. Ces traductions sont à considérer en liaison avec l'organisation discursive du texte, comme le montre l'exemple suivant ${ }^{11}$ :

Passons maintenant au cas où $\mathrm{A}$ est réduit...

Passons enfin au cas général. Notons que l'ensemble... Supposons donc $s>1$ et notons 墈 ...

Now let us consider the case in which A is reduced...

Let us finally consider the general case. Observe that... Suppose $s>1$ and write for...

On voit que les deux syntagmes impératifs traduits par «let us » correspondent à des étapes de la démonstration, alors que les impératifs traduits par un impératif en anglais correspondent, à l'intérieur d'une de ces étapes de la démonstration, à une série d'opérations de même niveau. Le traducteur a mis à profit la diversité des formes possibles de l'anglais pour marquer la diversité des valeurs discursives. Cet exemple nous paraît montrer très clairement comment, sur le plan énonciatif et discursif, le passage à l'anglais du discours mathématique offre ou impose les mêmes contraintes et les mêmes libertés à l'anglophone traduisant un texte français et au francophone rédigeant directement en anglais, et ce sont les implications de ce point que nous voudrions évoquer brièvement en conclusion de cette communication.

\section{Conclusion}

$\mathrm{Si}$, dans la tâche de relecture des "papiers" en anglais de ses collègues d'autres spécialités à laquelle l'angliciste au contact de la communauté scientifique se trouve souvent confronté, l'identification des fautes (essentiellement de grammaire) en violation des contraintes du système linguistique de l'anglais est aisée, il n'en va pas de même de toutes ces formulations dont l'acceptabilité paraît plus ou moins douteuse sans souvent qu'on y puisse mettre en évidence la responsabilité d'un élément précis. L'analyse contrastive, travaillant à partir d'un corpus traduit répondant à des critères de fiabilité, permet de faire apparaître des régularités dans les solutions et d'identifier certains des éléments déterminants. Ainsi, la traduction de "on», toujours difficile pour le francophone, fait également problème pour l'anglophone, le problème n'étant pas tant (sauf exception) l'acceptabilité de l'énoncé produit en anglais que la liberté de choix entre 
les possibles. Il est donc d'autant plus révélateur d'examiner ce qui peut conduire le locuteur natif à privilégier telle ou telle traduction, tâche qui consiste, pour le linguiste, à isoler les critères déterminants. Nous avons ainsi tenté de montrer, sur des points où le français et l'anglais ne disposent pas des mêmes possibilités morphologiques, quelques aspects de la diversité des paramètres qui entrent en jeu dans le phénomène même de l'activité langagière interlinguistique : garder la liberté de choisir ce que l'on veut dire et de le dire comme on veut malgré les contraintes inhérentes au système de la langue étrangère et au passage même d'un système à un autre.

\section{BIBLIOGRAPHIE}

Berland-Delépine, S. 1989. La grammaire anglaise de l'étudiant. Gap : Ophrys.

Bouscaren, Janine. 1991. Linguistique anglaise. Initiation à une grammaire de l'énonciation. Gap: Ophrys.

Bouscaren, Janine et Jean Chuquet. 1987. Grammaire et textes anglais. Guide pour l'analyse linguistique . Gap : Ophrys.

Chuquet, Hélène et Michel Paillard. 1989. Approche linguistique des problèmes de traduction anglaisfrançais. Gap : Ophrys.

Culioli, Antoine. 1991. Pour une linguistique de l'énonciation. Opérations et représentations. Gap : Ophrys.

Défourneaux, Marc. 1980. Do you Speak Science? Paris : Gauthier-Villars Bordas.

Faure, G. et J. Casanova. 1968. Nouvelle grammaire anglaise. Paris : Hatier.

Gardes-Tamine, Joëlle. 1990. La Grammaire 2/Syntaxe. Paris : Armand Colin.

Guillemin-Flescher, Jacqueline. 1981. Syntaxe comparée du français et de l'anglais. Problèmes de traduction. Gap : Ophrys.

Larreya, Paul et Claude Rivière. 1991. Grammaire explicative de l'anglais. Paris : Longman France.

Maingueneau, Dominique. 1981. Approche de l'énonciation en linguistique française. Paris : Hachette.

Petit, Michel. 1991. « Le génitif en 's, le génitif en of et la structure NøN avec les noms propres dans le discours mathématique en anglais ». Les Cahiers de l'APLIUT 10/4, 83-99.

Quirk, Randolph et al. 1972. A grammar of Contemporary English. Londres : Longman.

Wagner, R.L. et J. Pinchon. 1962. Grammaire du français classique et moderne. Paris : Hachette.

\section{NOTES}

1. On sait en effet que cette pratique est de plus en plus répandue parmi les universitaires et chercheurs français, singulièrement dans les disciplines scientifiques, où la diffusion des travaux et plus généralement l'accès à la communauté internationale passent nécessairement par 
l'anglais. Une large discussion s'engage sur ce sujet dans le débat qui suit l'exposé, plusieurs intervenants soulignant le rôle déterminant de la qualité linguistique de l'anglais dans l'acceptation ou le refus des "papiers" par les journaux scientifiques. Le rôle de conseil linguistique de l'enseignant d'anglais vis-à-vis de ses collègues d'autres disciplines apparaît à cet égard essentiel.

2. Cf. Maingueneau $1981: 32-33$.

3. Toute partie du discours affectée d'un intertitre générique et numérotée ("Théorème 5.2.2. ", «Lemme 3 », « Définition X-2 », etc.) semble pouvoir être a priori considérée comme de nature à présenter des caractéristiques de formulation stéréotypée (sur ce point cf. Petit 1991: 85) et relever plutôt de ce que nous appelons la fonction de démonstration. Ce point fait l'objet d'une discussion au cours du débat qui suit l'exposé. Il serait peut-être plus exact de parler de texte à contenu proprement mathématique. L'énoncé d'un théorème doit ainsi répondre à certaines conditions de bonne formation, qui garantissent sa validité mathématique et se traduisent dans son organisation linguistique. De la même façon, quelle qu'en soit l'organisation précise, toute section de texte intitulée "Démonstration » ou "Proof» est nécessairement orientée vers un résultat et fait nécessairement appel à des résultats antérieurs. Ceci se traduit là encore au niveau de l'organisation linguistique : «Montrons que... D'après..., on a... Or, il résulte de... que... D'où il s'ensuit que...». Ce que nous appelons fonction de commentaire comporte des interventions plus directes de l'auteur, des commentaires sur la facilité ou la difficulté de telle ou telle approche par exemple ou une explicitation de la façon dont il conduit le raisonnement («Dans ce chapitre, nous nous proposons de montrer... »). Il faudrait toutefois poursuivre plus précisément l'analyse car on peut trouver ces éléments de commentaire à l'intérieur d'une section de démonstration, même si leur statut différent est souvent indiqué par des procédés de mise en page tels que mise entre parenthèses, composition en caractères d'un corps inférieur, etc.

4. Nous entendons le terme "énonciatif " au sens où il est employé dans le cadre de la linguistique de l'énonciation d'Antoine Culioli (1991). L'application à l'anglistique en est notamment illustrée dans un certain nombre de travaux de Janine Bouscaren (Bouscaren \& Chuquet 1987 ; Bouscaren 1991).

5. Notre approche est contrastive en ce sens qu'elle utilise les données résultant de l'observation d'un corpus de textes traduits, suivant la méthodologie de l'analyse contrastive développée par Jacqueline Guillemin-Flescher (1981). Il est impératif à cet égard que la traduction réponde à certains critères de fiabilité, et notamment qu'elle soit due à un mathématicien anglophone. C'est le cas pour l'ensemble des traductions prises en considération dans cette communication.

6. On trouve par exemple une discussion détaillée de ce point dans Wagner \& Pinchon (1962: 294).

7. J. Gardes-Tamine signale que «ces pronominaux sont très productifs, en particulier dans la langue populaire et en argot: "se casser, se magner, se carapater, se trisser"... » (Gardes-Tamine 1990 : 87).

8. Cf. à cet égard Bouscaren \& Chuquet $1987: 167-179$.

9. Au cours du débat qui suit l'exposé, J. Coulardeau s'interroge sur l'utilisation privilégiée de « shall » pour l'expression de la référence future dans le discours mathématique. Nous proposons une explication qui pourrait tenir à un phénomène de surcorrection, hypothèse confirmée et développée par J.-L. Vidalenc qui fait état de ses propres observations sur le niveau stylistique des papiers scientifiques.

10. Serre, Jean-Pierre. Algèbres de Lie semi-simples complexes. New York: Benjamin, 1966 : 1-4 ; Complex Semisimple Lie Algebras. Trad. Jones, G.A. New York : Springer Verlag, 1987 : 3.

11. Samuel, Pierre. Théorie algébrique des nombres. Paris : Hermann, 1967, $2^{\mathrm{e}}$ éd. revue et corrigée 1971 : 79 ; Algebraic Theory of Numbers, Trad. Silberger, Alan J. Paris : Hermann 1970 et Londres : Kershaw, $1971: 65$. 


\section{RÉSUMÉS}

Analyse de linguistique contrastive visant à dégager certaines conditions spécifiques de passage à l'anglais du discours mathématique français, à la fois dans les cas de traduction par un anglophone d'un texte français existant et de rédaction directe en anglais par un francophone. Identification des contraintes linguistiques tenant aux différences de fonctionnement entre les deux langues, notamment à l'absence d'équivalents directs en anglais de configurations françaises récurrentes.

The aim of this paper, based on a contrastive linguistics analysis, is to highlight some of the specific conditions which characterize the translation into English of French mathematical texts or the writing in English of mathematical texts by French-speaking authors. It tries to identify the linguistic constraints, especially enunciative ones, due to the differences in the linguistic systems of French and English, particularly in the case of recurring French structures with no direct equivalent in English.

\section{INDEX}

Mots-clés : discours, énonciation, linguistique contrastive, mathématiques, traduction Keywords : contrastive linguistics, discourse, enunciation, mathematics, translation

\section{AUTEUR}

\section{MICHEL PETIT}

Université de Provence, Aix-Marseille 1, Département d'Etudes du Monde Anglophone. michel.petit@u-bordeaux2.fr 